\title{
BMJ Open Effects of preconditioning by nasal splint and mouth breathing on emergence delirium after functional endoscopic sinus surgery in Chinese adults: a study protocol for a randomised controlled trial
}

\author{
Hongjiao Xu (D) , ${ }^{1}$ Xiang Li, ${ }^{1}$ Bin Yang, ${ }^{2}$ Zhenyuan Shen, ${ }^{3}$ Weiwen Li, ${ }^{1}$ \\ Yachun Zhou, ${ }^{1}$ Jihong Jiang, ${ }^{1}$ Xia Chen, ${ }^{1}$ Yuyu Gu, ${ }^{1}$ Zhi Pei, ${ }^{1}$ Jinbao Li (D) ${ }^{1}$
}

To cite: $\mathrm{Xu} \mathrm{H,} \mathrm{Li} \mathrm{X,} \mathrm{Yang} \mathrm{B,} \mathrm{et} \mathrm{al.}$ Effects of preconditioning by nasal splint and mouth breathing on emergence delirium after functional endoscopic sinus surgery in Chinese adults: a study protocol for a randomised controlled trial. BMJ Open 2020;10:e033803. doi:10.1136/ bmjopen-2019-033803

- Prepublication history and additional material for this paper are available online. To view these files, please visit the journal online (http://dx.doi org/10.1136/bmjopen-2019033803).

Received 24 August 2019 Revised 09 July 2020 Accepted 22 July 2020

Check for updates

(c) Author(s) (or their employer(s)) 2020. Re-use permitted under CC BY-NC. No commercial re-use. See rights and permissions. Published by BMJ.

'Department of Anaesthesiology, Shanghai Jiaotong University First People's Hospital,

Shanghai, China

2Department of Anaesthesiology, Chongqing University Cancer Hospital, Chongqing, China ${ }^{3}$ Medical department, Mellon community health service center, Shanghai, China

Correspondence to

Dr Jinbao Li;

lijinbaoshanghai@163.com

\section{ABSTRACT}

Introduction Emergence delirium (ED) is a common adverse manifestation after general anaesthesia and may result in undesirable consequences. Its causes and mechanisms are diverse and complex, and it is still unavoidable in clinical work. There is a high incidence of ED after otorhinolaryngology surgery, which may result from the sudden loss of functional senses and discomfort of surgical organs. This study aims to test a non-invasive, non-drug treatment modality of nose clamping and mouthbreathing training before surgery to reduce ED. Methods and analysis This prospective randomised controlled trial (RCT) will include 200 patients who undergo functional endoscopic sinus surgery (FESS) at Shanghai General Hospital, China. Study participants will be randomly assigned in two groups with a 1:1 ratio. The pretreatment group (P-group) will receive an intervention by nasal splint and mouth-breathing training before surgery, while the control group (C-group) will not receive any intervention; following which both groups will undergo FESS under general anaesthesia in accordance with the same anaesthesia scheme. After surgery, we will perform a single-blinded assessment of ED occurrence with stratification. IBM SPSS Statistics V.20 statistical software will be used for statistical analyses. $A X^{2}$ test will be used to compare the two groups, and t-tests will determine the statistical significance of continuous variables.

Ethics and dissemination This RCT was designed in accordance with the principles of the Declaration of Helsinki and has been approved by the Ethics Committee of Shanghai General Hospital, ID: 2019KY039. We expect to release the original data in February 2022 on the ResMan original data sharing platform (IPD sharing platform) of the China clinical trial registry, which can be viewed at the following website:http://www.medresman.org.cn/pub/cn/ proj/projectshow.aspx?proj=6293.

Trial registration number ChiCTR1900024925

\section{INTRODUCTION}

Emergence delirium (ED or emergence agitation) is a well-known adverse phenomenon
Strengths and limitations of this study

- This randomised controlled trial (RCT) will employ non-invasive and non-pharmacological intervention.

- No blood or tissue samples will be obtained during this RCT.

- The outcomes will be evaluated by a single-blind method.

- We have enough patients for potential increases in the sample size without advertising for recruitment.

- The test performer and participants cannot be blinded to the intervention; this represents a limitation.

in the waking period of general anaesthesia (GA). It is described as a short-term state of confusion that occurs immediately after GA and lasts for 15-30 min. Clinically, the patients are perceived as 'waking' from GA, but with disorientation, hallucinations, hypersensitivity to external stimuli, restlessness and hyperactivity. ${ }^{1}$ The sudden occurrence of ED may result in serious outcomes such as; increased pain, bleeding, removal of the endotracheal tube, urinary catheter, drainage tube, sometimes even requiring additional medication and/or binding support. These may, in turn, increase the postanaesthesia care unit (PACU) time, and the medical and nursing costs.

Over the years, reports on the incidence of adult ED after GA have varied from $3.7 \%^{2}$ to $21.3 \% .^{3}$ Age, preoperative anxiety, postoperative pain, indwelling catheter, preoperative benzodiazepines, use of inhaled anaesthetics and the type of operation are some of the key factors instigating ED. ${ }^{13} 4$ Otorhinolaryngology surgery or surgeries involving the ear, nose and throat (ENT) are considered 
to be an independent risk factor for postoperative agitation. ${ }^{356}$ In 2006, Lepousé $e t ~ a l^{4}$ showed a $12 \%$ incidence of ED after ENT surgery in adults, while the overall ED incidence was $4.7 \%$. In a study of 2000 cases in 2010, Yu et $a \hat{l}$ reported an ED incidence of $55.4 \%$ after ENT surgery, and a total ED incidence of $21.3 \%$ irrespective of the surgery type.

After nasal surgery and during emergence from anaesthesia, ED occurs especially just after or during extubation or in the PACU. Patients who experience ED most often complain of difficulty in breathing. The literature points to this sudden suffocating discomfort as a potential cause of ED. ${ }^{6}$ However, the underlying mechanism is not completely understood, and this correlation requires to be confirmed further.

Functional endoscopic sinus surgery (FESS) is one of the most commonly performed nasal surgeries. Dissolvable foams are routinely placed in the nasal cavity after FESS for haemostasis, and to prevent postoperative adhesions. ${ }^{7}$ These foams can also cause mild sinus headache and discomfort during expansion in the nasal cavity. ${ }^{8}$ Absorbable foams do not require removal and begin to degrade at 24 hours, with most of the degradation occurring at 48 hours. However, during anaesthetic emergence, patients with nasal foams face a feeling of suffocation and are forced to breathe by mouth. Even without this absorbable foam, the pain in the nasal cavity caused by postoperative mucosal oedema and the surgical wound can lead to nasal congestion and breathing discomfort for a short duration, requiring forced oral breathing when the patient wakes up.

In general, anaesthesiologists often inform patients preoperatively about the nasal package or indwelling catheter, and the feeling of suffocation associated with it during the awakening period. This preoperative propaganda provides a certain psychological advantage and may help guard against ED, but the effect is not very ideal. According to a recent study on children, visual preconditioning with an eye mask 1 day before eye surgery effectively reduced ED, which was probably caused by visual disturbances on the affected side after surgery. ${ }^{9}$ This indicates that postoperative discomfort caused by sensory abnormalities of the target organ may contribute to ED. Thus, appropriate preoperative sensory preconditioning might effectively relieve ED. Herein, we describe a protocol for a randomised controlled trial (RCT) that aims to test a non-drug treatment modality for preventing ED after FESS.

\section{METHODS AND ANALYSIS}

\section{Trial objective}

In this study, we adopt the nasal splint and mouthbreathing training to simulate the sensory discomfort experienced after FESS. We test nasal splinting and mouth-breathing training as non-drug modalities, to study its influence on ED occurrence after FESS in adults.

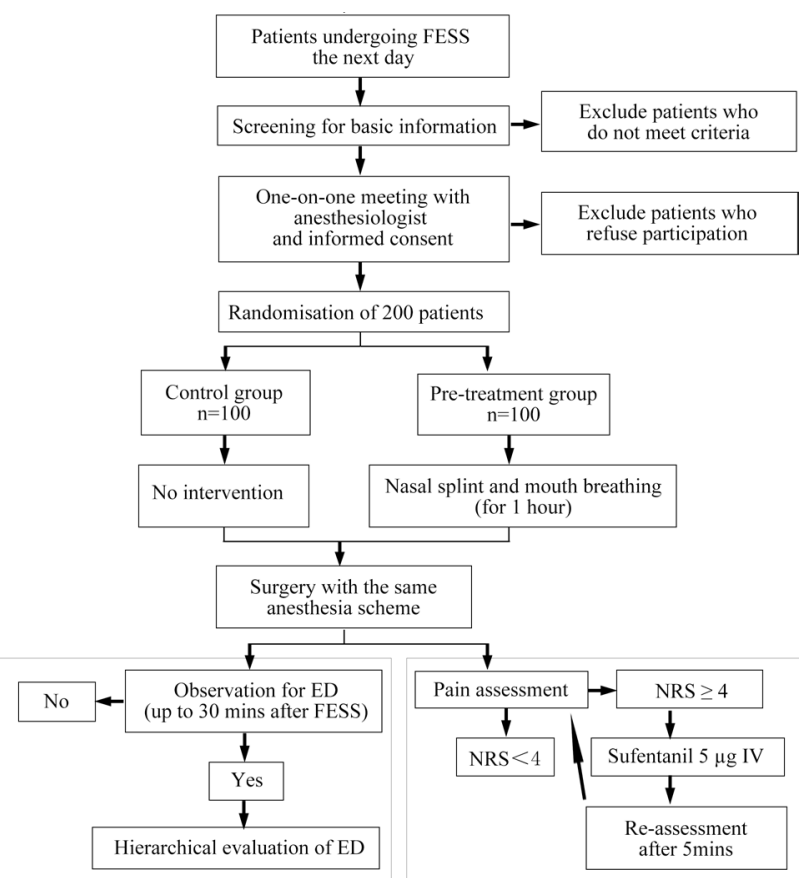

Figure 1 Flow chart of the trial design. ED, emergence delirium; FESS, functional endoscopic sinus surgery; NRS, Numerical Rating Scale.

\section{Trial design}

This protocol was prepared in accordance with the Standard Protocol Items: Recommendations for Interventional Trials reporting guidelines. ${ }^{10}$ This study intends to adopt the method of prospective RCT and use random numbers to divide all subjects meeting the inclusion criteria into the control group (C-group) and the pretreatment group (P-group). The nasal splint will be used as the intervention to train subjects' mouth breathing before FESS in the P-group, while the C-group will receive no intervention. The two groups will experience the same anaesthesia scheme (see below) and the same postoperative pain control scheme (see below). We will observe and evaluate the occurrence of ED in the two groups. A flowchart of the trial design is shown in figure 1.

\section{Anaesthesia scheme}

The subjects will not receive any preoperative medication. Once the patient enters the operating room, routine vital signs such as ECG, non-invasive blood pressure (NBP), oxygen saturation $\left(\mathrm{SpO}_{2}\right)$ and end-tidal carbon dioxide $\left(\mathrm{EtCO}_{2}\right)$ will be monitored. GA will be induced in all patients with midazolam $0.05 \mathrm{mg} / \mathrm{kg}$, propofol $2 \mathrm{mg} / \mathrm{kg}$, sufentanil $0.25 \mu \mathrm{g} / \mathrm{kg}$. After the loss of consciousness, the nasal splint will be removed in the P-group, and rocuronium $0.6 \mathrm{mg} / \mathrm{kg}$ given to both groups.

Endotracheal tube insertion will follow muscle relaxation. Using $60 \%$ oxygen $\left(\mathrm{O}_{2}\right)$ support, the $\mathrm{EtCO}_{2}$ will be maintained between 35 and $45 \mathrm{~mm} \mathrm{Hg}$. Desflurane at 0.8 and 1.0 minimum alveolar concentration (MAC) will be administered to maintain anaesthesia. Sufentanil 5-10 $\mu$ g will be given before the nasal endoscopic procedures according to the patients' body weight and 
circulatory state. Due to the short operation time of FESS, no additional intraoperative sufentanil administration is required. When the surgery duration exceeds 1 hour, or in the event of increasing heart rate and blood pressure rise due to light anaesthesia, an additional dose of 5-10 $\mu \mathrm{g}$ sufentanil will be given depending on the body weight and vital signs of the patient. Also, if the surgical wound is larger than normal, an additional $5 \mu \mathrm{g}$ sufentanil will be given before placing the nasal package. Neostigmine antagonist will be dosed based on the patients' muscle relaxation.

The extubation indicators will be tidal volume up to $5 \mathrm{~mL} / \mathrm{kg}$, and respiratory rate up to $12 / \mathrm{min}$, with obvious swallowing reflex, and $\mathrm{SpO}_{2}$ maintained at $95 \%$ or above after breathing air for $5 \mathrm{~min}$. Due to possible intraoperative procedures such as sympathetic stimulation caused by adrenalin infiltration in the nasal cavity; nimodipine and esmolol will be used to control NBP and heart rate within the normal range.

\section{Pain assessment and control}

Postoperative PACU monitoring will include ECG, NBP, $\mathrm{SpO}_{2}$ and $\mathrm{EtCO}_{2}$. Pain assessment will be carried out by a trained anaesthesia nurse blinded to grouping information. The Numerical Rating Scale (NRS) will be used for pain assessment every 5 min immediately after extubation. The pain scale ranges from 0 to 10 , with 0 representing no pain (painless) and 10 representing the most pain. Pain control will be performed by the anaesthesiologist in the PACU. For scores of 4 or above, sufentanil 3-5 $\mu \mathrm{g}$ will be administered by intravenous injection, with the NRS reassessed 5 min later to keep the pain below 4 .

\section{Participants and recruitment}

This RCT will be conducted at the Shanghai General Hospital, China. Over 30 patients undergo FESS at the Shanghai General Hospital every week. Thus, we will be able to achieve the sample size without advertising for recruitment. All patients planned for elective FESS at the Shanghai General Hospital will be screened according to the inclusion and exclusion criteria the day before surgery.

Patients meeting the inclusion criteria will be interviewed the afternoon before surgery by a trained anaesthesiologist in the ward. Following the one-on-one meeting, the patient will decide whether to participate in the study based on the general information provided. If the patient decides to participate, he/she will sign the informed consent in duplicate, which will be retained by both the patient and the researcher.

\section{Inclusion criteria}

The inclusion criteria will be:

1. Patients undergoing FESS surgery in Shanghai General Hospital.

2. Age over 18 years.

3. American Society of Anaesthesiologists Classification (ASA) class I-II, no serious cardiovascular disease.
4. Preoperative liver and kidney function within the normal range.

5. Those who, through publicity and mobilisation, know about the trial and voluntarily participate by signing the informed consent.

\section{Exclusion criteria}

Patients with any one of the following criteria will be excluded:

1. ASA grade III-V, severe cardiovascular disease or poor blood pressure control.

2. History of mental illness.

3. History of sedative or antipsychotic drug use (neuroinhibitors, anxiolytics, antidepressants, benzodiazepines) for any reason.

4. History of neurological diseases (cerebral infarction, transient ischaemic attack, Parkinson's disease, mental retardation, craniocerebral injury).

5. Nasal malformation, history of nasal trauma, implantation of nasal prosthesis.

6. Surgical complications (socket perforation, massive bleeding requiring blood transfusion or reoperation, cerebrospinal fluid leakage, eyelid emphysema, intraorbital bleeding, meningitis, partial loss of vision, double vision, blindness, death). ${ }^{11}$

\section{Randomisation}

The participants will be divided into C-group and P-group in a total ratio of $1: 1$ by means of simple randomisation. The random number sequence (one as C-group and two as P-group) was generated by computer using the SPSS software, IBM SPSS Statistics V.20 (IBM).

\section{Sample size calculation}

PASS V.15.0 (NCSS) was used to calculate the sample size. According to the incidence of $\mathrm{ED},{ }^{3} \mathrm{p}<0.05$ is considered significant, $\alpha=0.05, \beta=0.1$, then $\mu \alpha=1.96, \mu \beta=1.28$. We set the OR as 3.0 based on our preliminary experimental results and a previous study. ${ }^{12}$ The effective sample size of each group was 82, with a total of 164 patients. Considering the possible loss (surgery cancelled, patients refused to participate in the process, etc), we decided to recruit 200 patients (100 for each group) to ensure a sufficient sample size.

\section{Blinding}

The patients' information will be collected 1 day before surgery by a data collector (the study leader), and only those who meet the requirements for the research will be disclosed to a group divider. The night before the surgery, the group divider will group the patients according to the random number sequence. This list will be handed over to the test performer the morning of the surgery day. The test performer will execute the preconditioning and anaesthesia in accordance with the original order of surgery appointment.

The collector tells only the participants' name but nothing to the group divider the night before surgery. The test performer and participants will be given the group 
information the surgery day morning. The data collector, group divider and test performer are three different but constant people in this research, and roles may not be repeated. The data collector and group divider will not participate in any second-day research. The random number sequence is retained by the group divider and the study leader for backup. In addition, the evaluator of outcome indicators and the pain controller do not know the group information at all.

We will assess the outcome indicators using a singleblind method. The evaluator of outcome indicators and the pain controller will not know the grouping information during the whole research process.

\section{Interventions}

Patients in P-group will receive nasal splint for preoperative nasal intervention and training of mouth breathing. Patients will arrive at the preanaesthesia room 1 hour before surgery and be monitored with $\mathrm{SpO}_{2}$ and NBP. The nasal splint will be used to clamp the subnasal cartilage in front of the nasal cavity to ensure that patients cannot breathe through the nose, and patients will be instructed to breathe through the mouth. The mouth-breathing training is considered successful when $\mathrm{SpO}_{2}, \mathrm{HR}$ and NBP are close to the baseline. The patients will then wait quietly and adapt to the nasal splint state. The nose clip will not be removed until the anaesthesia is induced, and the patients lose consciousness in the operating room.

Patients in C-group will not take any intervention measures and will not be informed of the possible nasal congestion and discomfort after surgery. If patients of different groups are waiting for surgery in the preanaesthesia room at the same time, isolation will be employed so that they do not share information.

If a patient in P-group cannot tolerate the nasal splint state or his $/$ her $\mathrm{SpO}_{2}$ is below $95 \%$ for 5 min, researchers will end the intervention and give the patient $4 \mathrm{~L} / \mathrm{min}$ $\mathrm{O}_{2}$ until $\mathrm{SpO}_{2}$ returns to normal. Moreover, this patient will be removed from this study (not included in the C-group).

\section{Outcome measurements}

The primary outcome of this study is the observation of $\mathrm{ED}$, and the secondary outcome is the hierarchical evaluation of ED. Assessments will be performed in both groups by the same anaesthesia nurse and recorded in detail.

\section{Primary outcomes}

\section{Observation of ED assessment}

Following postoperative extubation to any time within 30 min after FESS, the Riker Sedation-Agitation scale will be used (table 1). ${ }^{13}$ A score of $5-7$ points indicates the presence of ED, and a score of 1-4 indicates no ED.

\section{Secondary outcomes}

Hierarchical evaluation of ED

ED will be defined as combative behaviour, thrashing and hyperactive motor behaviour (table 2 ) ${ }^{14}$ If the above behaviour occurs in response to powerful stimulation such

\begin{tabular}{|c|c|c|}
\hline Score & $\begin{array}{l}\text { Emergence } \\
\text { behaviour }\end{array}$ & Clinical examples \\
\hline 7 & $\begin{array}{l}\text { Dangerous } \\
\text { agitation }\end{array}$ & $\begin{array}{l}\text { Pulling at endotracheal (ET) tube, } \\
\text { trying to remove catheters, climbing } \\
\text { over bed rail, striking at staff, } \\
\text { thrashing side-to-side. }\end{array}$ \\
\hline 6 & $\begin{array}{l}\text { Very } \\
\text { agitated }\end{array}$ & $\begin{array}{l}\text { Does not calm despite frequent verbal } \\
\text { reminding of limits; requires physical } \\
\text { restraints, biting ET tube. }\end{array}$ \\
\hline 5 & Agitated & $\begin{array}{l}\text { Anxious or mildly agitated, attempting } \\
\text { to sit up, calms down to verbal } \\
\text { instructions. }\end{array}$ \\
\hline 4 & $\begin{array}{l}\text { Calm and } \\
\text { cooperative }\end{array}$ & $\begin{array}{l}\text { Calm, awakens easily, follows } \\
\text { commands. }\end{array}$ \\
\hline 3 & Sedated & $\begin{array}{l}\text { Difficult to arouse, awakens to verbal } \\
\text { stimuli or gentle shaking but drifts off } \\
\text { again, follows simple commands. }\end{array}$ \\
\hline 2 & Very sedated & $\begin{array}{l}\text { Arouses to physical stimuli but } \\
\text { does not communicate or follow } \\
\text { commands may move spontaneously. }\end{array}$ \\
\hline 1 & Unarousable & $\begin{array}{l}\text { Minimal or no response to noxious } \\
\text { stimuli, does not communicate or } \\
\text { follow commands. }\end{array}$ \\
\hline
\end{tabular}

as suctioning phlegm, but stops with stimulus removal, the assessment is mild. If ED is observed without stimulation and lasts for at least $5 \mathrm{~min}$, and does not require medical intervention, it is rated as moderate. It is rated as severe if it lasts for at least $5 \mathrm{~min}$ and must be controlled with medication and/or physical restraint.

\section{Data and capture method}

Data will be collected both from the hospital information system of the Shanghai General Hospital and by a oneon-one interview by the person in charge of the experiment (data collector/study leader).

According to relevant factors in the literature, preoperative information collected will include age, gender, ASA classification, disease severity (CT of the paranasal sinuses and nasal endoscopy, table 3), nasal congestion history and smoking. In the preanaesthesia room, heart

\section{Table 2 Degree of ED*}

Mild $\quad$ ED in response to powerful stimulation such as suctioning phlegm, but reverses on stimulus removal.

Moderate ED without stimulation, lasting for at least $5 \mathrm{~min}$, but does not require intervention of the attending staff.

Severe ED lasting for at least $5 \mathrm{~min}$ and had to be controlled by drugs and/or a physical restraint.

*Defined as combative behaviour, thrashing, and hyperactive motor behaviour.

ED, emergence delirium. 
Table 3 Preoperative rhinoscopy and CT of sinuses

\begin{tabular}{ll}
\hline Nasal endoscopic appearance & CT scan sinus \\
\hline Nasal polyps & Maxillary sinus \\
Nasal oedema & Anterior ethmoid \\
Nasal secretion & Posterior ethmoid \\
& Sphenoid sinus \\
& Frontal sinus \\
& Ostiomeatal complex \\
\hline
\end{tabular}

rate, $\mathrm{SpO}_{2}$, NBP before and after the intervention, and the intervention effect will be recorded. The following information will be collected during the surgery: operation time, anaesthesia time, intraoperative medication, changes in vital signs, absorbable foam filling site, presence or absence of indwelling catheter or drainage. Postoperative information collected will include: ED assessment, hierarchical evaluation of ED, pain assessment and medication administration within 30 min after surgery, retention of tracheal intubation, PACU duration, PACU medication and any other relevant information.

\section{Data and safety monitoring board}

To ensure the integrity and authenticity of the RCT and protect the participants' privacy, we will set up a data and safety monitoring board (DSMB). The DSMB is an independent panel of experts, each with at least 10 years of clinical experience. The panel will not participate in the implementation of this study. They will evaluate the eligibility of the researchers before the study has begun. They will regularly review the scientific and ethical standards of the RCT and examine the validity of the data analysis. They also have access to the interim results and make the final decision to terminate the trial. The DSMB will be developed in accordance to the Operational Guidelines for the Establishment and Functioning of DSMB of WHO and be responsible for data evaluation during the study period.

\section{Data statement}

All the data will be eventually collected and sent to the person who in charge of the study, who will keep it online on a secure server (baidu cloud disk, https://pan.baidu. com/). Only the study leader will have access to all the data. If other researchers want to obtain relevant data of this study, they can contactXL or HX by email when the study is finished.

\section{Statistical analysis}

IBM SPSS Statistics V.20 statistical software will be used to analyse data from the two groups. Continuous variables of normal distribution will be expressed as mean $\pm \mathrm{SD}$, others of non-normal distribution will be expressed as median and IQR. Categorical variables will be described as number $(\mathrm{n})$ and percentage $(\%)$. A $\mathrm{X}^{2}$ test will be used to compare the two groups, and t-tests will determine the statistical significance of continuous variables.
Binary logistic regression analyses will be used to estimate the independent impacts of intervention (preoperative nasal intervention and training of mouth breathing) on outcome (occurrence of ED) adjusted for other potential factors. All significance tests will be two sided, and a two-tailed $p$ value of 0.05 or less will indicate statistical significance.

\section{Harms}

The intervention in this study is the nasal splint; a noninvasive and non-pharmacological approach to prevent ED. On top of this, no blood or tissue samples will be obtained during this RCT. This will minimise the harm and discomfort brought to patients by the study.

\section{Ethics and dissemination}

This RCT was designed in accordance with the principles of the Declaration of Helsinki and has been approved by the Ethics Committee of Shanghai General Hospital (ID: 2019KY039). The approval is valid for 12 months, and the ethics office will review the study every 12 months and issue an ethical approval for the next 12 months. If there is any change in the protocol, we will submit a modification application again and suspend the study until it is approved.

All the participants will provide informed consent (see online supplementary file 1) prior to commencing their involvement in the study. They will have the opportunity to contact one of the researchers in any form, or at any time before or after the RCT initiation. They will be able to raise any questions or concerns they might have about the study or procedures. They will also have the right to withdraw from the study at any point.

We expect to release the original data in February 2022 on the ResMan original data sharing platform (IPD sharing platform) of China clinical trial registry (http:// www.medresman.org.cn/pub/cn/proj/projectshow. aspx?proj=6293).

\section{Patients and public involvement}

The patients or the public are not involved in the design, conduct, reporting or dissemination of our research, and no attempt will be made to assess the burden of the intervention on the patients themselves.

\section{DISCUSSION}

ED is described as a short-term state of confusion, which occurs immediately after GA and lasts for $15-30 \mathrm{~min} .{ }^{1}$ It is a transient phenomenon, and if it recurs, it is known as postoperative delirium (POD); this is one of the key points in identifying ED and POD. ${ }^{15}$ We have, therefore, set our observation time to $30 \mathrm{~min}$ after surgery. Clinically, the symptoms of ED are relieved after the doctor removes the stimulation or comforts the patient and do not recur. In our study, whenever ED may cause possible self-harm to patients (the most likely is to remove the external gauze on the nose), we will immediately take measures such as 
soothing, physical restraint or administration of sedatives; these will be recorded.

This article describes the design and protocol of a prospective RCT, that aims to reduce ED after GA in adults. Another study from Egypt ${ }^{12}$ using external nasal compression also confirmed the effectiveness of such interventions. However, it had a small sample size (60 participants in three groups) and a wide range of surgical types (septoplasty, septorhinoplasty and FESS). In contrast, our study first emphasises mouth-breathing training as an intervention after the nasal splintand has set the intervention time at 1 hour to ensure adequate breathing adaptation time. Second, owing to the large number of FESS surgeries in our hospital, we can ensure an adequate sample size, and can select the same operation (FESS) to reduce errors caused by the type of surgery. The results may indicate a non-invasive, non-pharmacological approach to reduce ED; this may benefit both patients and clinical practice.

\section{Trial status}

Medical ethical approval was obtained on 17 July 2019, and clinical trial registration was approved on 3 August 2019. The first participant was enrolled on 18 September 2019 and by 27 December 2019, a total of 73 participants had been enrolled. The study has been suspended since then owing to the first-level response launched by the Shanghai government to th enew coronavirus epidemic (COVID-19), which is a major public health emergency. The study was resumed on 9 May 2020 when the emergency response level in Shanghai was lowered to level 3.

Acknowledgements We would like to thank all the supervisors and review boards of this study, and Shanghai General Hospital for providing study site and cases, and Editage (www.editage.cn) for English language editing.

Contributors $\mathrm{JL}$ initially formulated the research ideas and helped $\mathrm{HX}$ to complete the manuscript. $\mathrm{HX}$ is in charge of the study, wrote the manuscript and completed the ethical approval and trial registration, who also will be the data collector, and perform the one-on-one interview before study. XL will execute the intervention in P-group, and performan anaesthesia management for all patients. BY further modified the manuscript and revised the statistical analysis. ZS completed the registration of our study on the ResMan original data sharing platform (IPD sharing platform) and will be responsible for uploading all the data of this study. WL calculated the sample size, and will be responsible for the grouping work. YZ and $\mathrm{JJ}$ will be responsible for data analysis. XC will perform the pain control in PACU. YG and her assistant ZP will be responsible for the assessments after surgery, including the observation of ED and the hierarchical evaluation of ED and pain assessment.

Funding The Shanghai Songjiang District Programmes for Science and Technology Development (18sjkjgg57). Basic science and advanced technology foundation of Chongqing Science and Technology Commission (cstc2016jcyjA0158). Performance incentive and guidance project of scientific research institutes of Chongqing Science and Technology Commission (cstc2018jxj|130028). High-level Medical Reserved Personnel Training Project of Chongqing (2019GDRC017). Foundation of Chongqing Health and Family Planning Commission (ZY201702036).

Competing interests None declared.

Patient consent for publication Not required.

Provenance and peer review Not commissioned; externally peer reviewed.

Open access This is an open access article distributed in accordance with the Creative Commons Attribution Non Commercial (CC BY-NC 4.0) license, which permits others to distribute, remix, adapt, build upon this work non-commercially, and license their derivative works on different terms, provided the original work is properly cited, appropriate credit is given, any changes made indicated, and the use is non-commercial. See: http://creativecommons.org/licenses/by-nc/4.0/.

\section{ORCID iDs}

Hongjiao Xu http://orcid.org/0000-0001-7483-4516

Jinbao Li http://orcid.org/0000-0001-5582-5737

\section{REFERENCES}

1 Munk L, Andersen LPH, Gögenur I. Emergence delirium. J Perioper Pract 2013;23:251-4.

2 Munk L, Andersen G, Møller AM. Post-anaesthetic emergence delirium in adults: incidence, predictors and consequences. Acta Anaesthesiol Scand 2016;60:1059-66.

3 Yu D, Chai W, Sun X, et al. Emergence agitation in adults: risk factors in 2,000 patients. Can J Anaesth 2010;57:843-8.

4 Lepousé C, Lautner CA, Liu L, et al. Emergence delirium in adults in the post-anaesthesia care unit. Br J Anaesth 2006;96:747-53.

5 Voepel-Lewis T, Malviya S, Tait AR. A prospective cohort study of emergence agitation in the pediatric postanesthesia care unit. Anesth Analg 2003;96:1625-30.

6 Eckenhoff JE, Kneale DH, Dripps RD. The incidence and etiology of postanesthetic excitment. A clinical survey. Anesthesiology 1961;22:667-73.

7 Berlucchi M, Castelnuovo P, Vincenzi A, et al. Endoscopic outcomes of resorbable nasal packing after functional endoscopic sinus surgery: a multicenter prospective randomized controlled study. Eur Arch Otorhinolaryngol 2009;266:839-45.

8 Szczygielski K, Rapiejko P, Wojdas A, et al. Use of CMC foam sinus dressing in FESS. Eur Arch Otorhinolaryngol 2010;267:537-40.

9 Lin Y, Shen W, Liu Y, et al. Visual preconditioning reduces emergence delirium in children undergoing ophthalmic surgery: a randomised controlled trial. Br J Anaesth 2018;121:476-82.

10 Chan A-W, Tetzlaff JM, Altman DG, et al. Spirit 2013 statement: defining standard protocol items for clinical trials. Ann Intern Med 2013;158:200-7.

11 Stammberger $\mathrm{H}$. The evolution of functional endoscopic sinus surgery. Ear Nose Throat J 1994;73:451-5.

12 Kasem A, Abdelkader A. Preoperative external nasal compression: does it decrease emergence agitation after nasal surgery? AinShams J Anaesthes 2016;9:593-7.

13 Riker RR, Picard JT, Fraser GL. Prospective evaluation of the sedation-agitation scale for adult critically ill patients. Crit Care Med 1999;27:1325-9.

14 Cohen IT, Hannallah RS, Hummer KA. The incidence of emergence agitation associated with desflurane anesthesia in children is reduced by fentanyl. Anesth Analg 2001;93:88-91.

15 Aldecoa C, Bettelli G, Bilotta F, et al. European Society of Anaesthesiology evidence-based and consensus-based guideline on postoperative delirium. Eur J Anaesthesiol 2017;34:192-214. 